\title{
Generalized Building Polygon Partitioning for Automatic Generation of 3-D Building Models
}

\author{
Sugihara Kenichi ${ }^{1}$, Hayashi Yoshitugu ${ }^{2}$
}

\begin{abstract}
Building polygons are buildings' contours on the digital map stored by GIS. Based on building polygons, we are proposing a GIS and CG integrated system to generate 3-D building models automatically. A 3-D urban model is the important information infrastructure that can be utilized in several fields, such as landscape evaluation, urban planning, disaster prevention simulation, etc. To realize a 3-D urban model, it is important to generate building models efficiently. However, in order to realize a 3-D urban model, time consuming process is necessary to acquire the spatial data and to design the models. In order to automate the laborious process, the system aims at creating 3-D building models automatically from building polygons. If the building polygon with more than 4 vertices has right angle only, then it can be divided in rectangles. We propose the useful polygon expression where the building polygon can be expressed as a set of its edges' turning direction (RL expression) and that decides from which vertex a dividing line (DL) is drawn.
\end{abstract}

Keywords : $\quad$ 3-D Urban Model, Automatic Generation, CG, GIS, polygon partitioning

\section{INTRODUCTION}

When simulating a real city by a 3-D CG urban model, buildings are major objects in the model. A 3-D urban model as shown in Fig.2 is an important information infrastructure that can be utilized in several fields, e.g., landscape evaluation and city planning, architecture, disaster prevention simulation, tourism, and many other business practices. In addition, disclosure of information about public projects such as city planning or historic buildings restoration to the public in order to encourage their participation is a new application area where a 3-D city model can be of great use. The importance of disclosure of information has become greater and greater in order to carry out public projects smoothly. In Japan, public agencies publicize the results of excavations or urban planning by showing floor plans and side views and by explaining with papers difficult to understand. To facilitate "Public Involvement", 3-D models simulating a real city by a 3-D CG can be of great use.

However, in order to realize a 3-D city model, enormous time and labor has to be consumed to acquire the spatial data and to design the models. For example, when manually modeling a house with roofs by Constructive Solid Geometry (CSG), one must follow these laborious modeling steps: 1) generation of primitives of appropriate size, such as box, prism or polyhedron that will form parts of a house 2) boolean operation among these primitives to form the shapes of a roof and a house body 3 ) rotation of parts of a house 4) positioning of parts of a house 5) texture mapping onto these parts.

So, we proposed GIS (Geographic Information System) and CG integrated system that automatically generates $3-D$ building models from building polygons on a digital map shown in Fig. ${ }^{1)}$. Building polygons are buildings' contours on a digital map stored by GIS. Most building polygons' edges meet at right angle (orthogonal polygon).

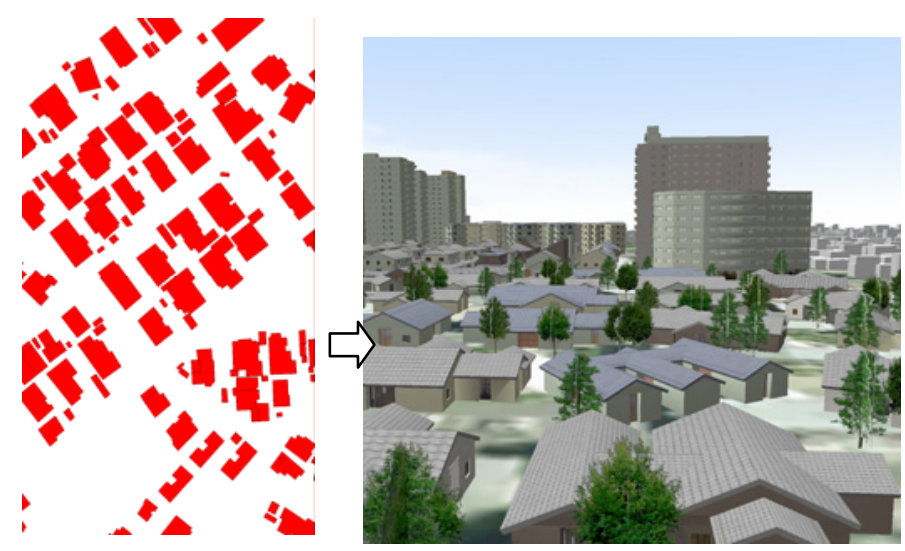

Fig. 1 Building polygons on a digital map generated 3-D urban model
Fig.2 An automatically

1) Member of JSCE, Dr.Eng., Professor, Faculty of Business Administration, Gifu Keizai University (Kitagata chou 5-50 Ogaki city Gifu pref. 503-8550, Japan)

2) Member of JSCE, Dr.Eng., Professor, Graduate School of Environmental Studies, Nagoya University ( Furou-chou Chikusa-ku, Nagoya 464-8603, Japan) 
A complicated orthogonal polygon can be partitioned into a set of rectangles. After partitioning, the integrated system is placing rectangular roofs and building bodies on these rectangles. In order to partition an orthogonal polygon, we proposed a useful polygon expression in deciding from which vertex a dividing line (DL) is drawn. In this paper, we propose a scheme of prioritizing the DL that forms a branch roof, based on the length of the DL.

\section{Related Work}

Since a 3-D urban model is an important information infrastructure that can be utilized in several fields, the researches on generation of 3-D urban models are in full swing. In many generation processes, the sources of 3-D building models are 2-D digital maps stored and administrated by GIS. 2-D digital maps are made by map production companies. Building polygons on a digital map are drawn manully with a digitizer, depending on the aerial photos. In the case of city planning, an urban designer may follow the traditional way of drawing building polygons for the future layout of a city.

Building polygons can be extracted from aerial images by an image recognition software. Aerial images are also important sources of 3-D building models. However, due to the complexity of natural scenes and the lack of performance of image recognition software, fully automated methods cannot guarantee results stable and reliable enough for practical use ${ }^{2)}$.

Recently, many approaches for automated and semi-automated extraction and modeling of buildings from aerial images have been proposed 3), 4), 5).

Gruen and Wang ${ }^{2)}$ introduced a semi-automated topology generator for 3-D building models: CC-Modeler. Feature identification and measurement with aerial images is implemented in manual mode. During feature measurement, measured 3-D points belonging to a single object should be coded into two different types according to their functionality and structure: boundary points and interior points. After these manual operations, the faces are defined and the related points are determined. Then the CC-Modeler fits the faces jointly to the given measurements in order to form a 3-D building model.

Suveg and Vosselman 4) presented a knowledge-based system for automatic 3D building reconstruction from aerial images. The reconstruction process starts with the partitioning of a building into simple building parts based on the building polygon provided by 2D GIS map. If the building polygon is not a rectangle, then it can be divided into rectangles. A building can have multiple partitioning schemes. To avoid a blind search for optimal partitioning schemes, the minimum description length principle is used. This principle provides a means of giving higher priority to the partitioning schemes with a smaller number of rectangles. Among these schemes, optimal partitioning is 'manually' selected. Then, the building primitives of CSG representation are placed on the rectangles partitioned.

These proposals and systems will provide us with a primitive 3D building model with accurate height, length and width, but without details such as windows, eaves or doors. The research on 3D reconstruction is concentrated on reconstructing the rough shape of the buildings neglecting details on the facades such as windows, etc ${ }^{6}$. On the other hand, there are some application areas such as urban planning and disaster prevention simulation where the immediate creation and modification of many plausible building models is requested to present the alternative 3-D urban models.

When generating these models, automatic modeling of many future buildings is to be desired. In these models, approximate 3D building models with details are necessary to encourage the public involvement so that people in general can recognize them as buildings of alternative urban plans. Usually and traditionally, urban planners design the future layout of a town by drawing a map. So, it is convenient for urban planners who draw maps if the map can immediately be converted into a 3-D urban model. In our research, we aim at creating 3-D urban models, especially building models automatically from 2D building contours on a digital map. The system automatically generates a 3-D urban model so quickly that it meets the urgent demand to realize another alternative plan. 


\section{FLOW OF THE AUTOMATIC GENERATION SYSTEM}

The automatic generation system consists of GIS application (ArcGIS, ESRI Inc.), GIS module and CG module as shown in Fig 3. The source of a 3-D urban model is a digital residential map that contains building polygons linked with attributes data such as the number of stories, the type of roof.

The GIS module pre-processes building polygons on the digital map. Pre-process includes filtering out 'short edge' (short edge that is between long edges of almost the same direction) and unnecessary vertices of a building polygon, dividing a building polygon whose edges meet at right angles ( orthogonal polygon) into a set of rectangles, generating inside contours to form walls and glasses of a building and exporting the coordinates of polygons' vertices and attributes of buildings. The attributes of buildings consist of the number of stories, the image code of roof, wall and the type code of roof (flat, gable roof, hipped roof, gable roof with longer width, gambrel roof, shed roof and penthouse). The GIS module has been developed using 2-D GIS software components (Map0bjects, ESRI).

The CG module receives the pre-processed data that the GIS module exports, generating 3-D building models. CG module has been developed using Maxscript that controls 3-D CG software (3D Studio VIZ, Autodesk Inc). In case of modeling a building with roofs, the CG module follows these laborious steps: 1) generation of primitives whose size are determined by the preprocessed data

2) boolean operation among these primitives to form the shapes of the roof, the building body, etc. 3) rotation of parts of the building 4) positioning of parts of the building 5) texture mapping to these parts according to the attribute received 6) copying the 2nd floor to form the 3rd floor or more in case of building higher than 3 stories.

\section{GIS MODULE}

\section{(1) Proposed Polygon Expression}

At map production companies, many technicians are drawing digital residential maps. The building polygons shown in Fig. 1 on the residential map are drawn manually with digitizer, depending on the aerial photos. The map shows that most building polygons are orthogonal polygon. An orthogonal polygon can be replaced by a combination of rectangles. 3D building models are placed on these rectangles by CSG. When following edges of a polygon clockwise, an edge turns to the right or to the left by 90 degrees. So, it is possible to assume that an orthogonal polygon can be expressed as a set of its edges' turning direction. So, we proposed a useful polygon expression ( RL expression ) in specifing the shape pattern of an orthogonal polygon ${ }^{1)}$. For example, a polygon with 36 vertices shown in Fig. 4 is expressed as a set of its edges' turning direction; LLRRRLLRRRLLLRRRRLLRLRRLLRRLRLLRRRRL where $\mathrm{R}$ and $\mathrm{L}$ mean a change of an edge' s direction to the right and to the left, respectively. The number of shapes that a polygon can take depends on

\begin{tabular}{|c|c|c|}
\hline $\begin{array}{l}\text { Building } \\
\text { Polygons on 2-D } \\
\text { Digital Maps }\end{array}$ & $\begin{array}{l}\text { GIS } \\
\text { Application } \\
\text { ( ArcGIS ) } \\
\text { *Building } \\
\text { Polygons on 2-D } \\
\text { Digital Maps } \\
\text { *Attribute for } \\
\text { 3-D model such } \\
\text { as number of } \\
\text { stories, type of } \\
\text { roof, image code } \\
\text { for mapping to } \\
\text { roof and wall }\end{array}$ & $\begin{array}{l}\text { GIS Module } \\
\text { ( VB using } \\
\text { MapObjects, ESRI } \\
\text { Inc) } \\
\text { *Dividing an } \\
\text { orthogonal } \\
\text { polygon into } \\
\text { rectangles } \\
\text { *Contour } \\
\text { Generation } \\
\text { *Filtering out } \\
\text { noise edges, } \\
\text { unnecessary } \\
\text { vertices }\end{array}$ \\
\hline
\end{tabular}

\section{CG Module}

(MaxScript that controls 3D Studio VIZ)

*Generating 3-D models based on preprocessed data

*Rotating and positioning 3D models

*Automatic texture mapping onto 3D models

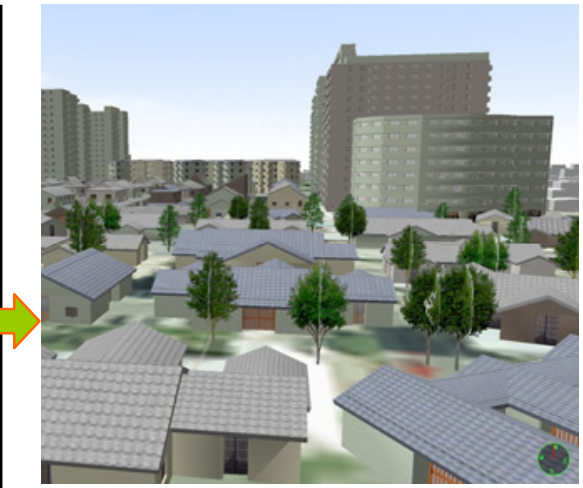

Fig. 3 Flow of Automatic Generation of 3D Building Models 
the number of vertices that a polygon has. CG module receives the pre-processed data that the GIS module exports, generating 3-D building models.

By the formula of circular permutation, we can calculate the number of the shape patterns that a polygon may take. In the case of a polygon with 6 vertices (6 vertices polygon), the edges' direction set of the polygon is LRRRRR. Since the left turn edge appears only once in 6 vertices polygon, the shape pattern is unique. In the case of an 8 vertices polygon, the following four kinds of polygon shape pattern are possible: LLRRRRRR, LRLRRRRR, LRRLRRRR, LRRRLRRR. LRRRLRRR has a reiteration pattern of ' LRRR' . Four cases can be calculated by the following formula of circular permutation.

As for 10 and 12 vertices polygon, the number of shape pattern is also calculated by the same formula. In proportion to the number of vertices, the number of shape pattern will increase by geometric progression.

(1) 8 vertices polygon:

No reiteration pattern reiteration patterns

$$
\left(\frac{8 !}{6 ! 2 !}-\frac{4 !}{3 ! 1 !}\right) \div 8=3 \quad \frac{4 !}{3 ! 1 !} \div 4=1
$$

Total number of cases: $3+1=4$ cases

(2) 10 vertices polygon:

$$
\frac{10 !}{7 ! 3 !} \times \frac{1}{10}=12 \text { cases }
$$

(3) 12 vertices polygon:

3 reiteration patterns

$$
\frac{3 !}{2 ! !} \div 3=1 \quad\left(\frac{6 !}{4 ! 2 !}-\frac{3 !}{2 !}\right) \div 6=2
$$

No reiteration pattern

$$
\left(\frac{12 !}{8 ! 4 !}-\frac{6 !}{4 ! 2 !}\right) \div 12=40
$$

Total number of cases: $1+2+40=43$ cases

The advantage of this RL expression is as follows.

1) RL expression specifies the shape pattern of an orthogonal polygon without regard to the length of its edges.

2) This expression decides from which vertex a dividing line (DL) is drawn.

\section{(2) How to Divide A Polygon}

A complicated orthogonal polygon can be partitioned into a set of rectangles. After partitioning, the integrated system is placing rectangular roofs and box-shaped building bodies on these rectangles. In order to partition an orthogonal polygon, we proposed RL expression in deciding from which vertex a dividing line (DL) is drawn ${ }^{1)}$.

Since the interior angle of a 'L' vertex is 270 degrees, two DLs can be drawn from a 'L' vertex. Figure 4 (down) shows an orthogonal polygon with possible DLs shown as dotted lines and DLs that are selected to be implemented a partition, shown as thick dotted lines.

In our proposal, among many possible DLs, the DL that satisfies the following conditions is selected
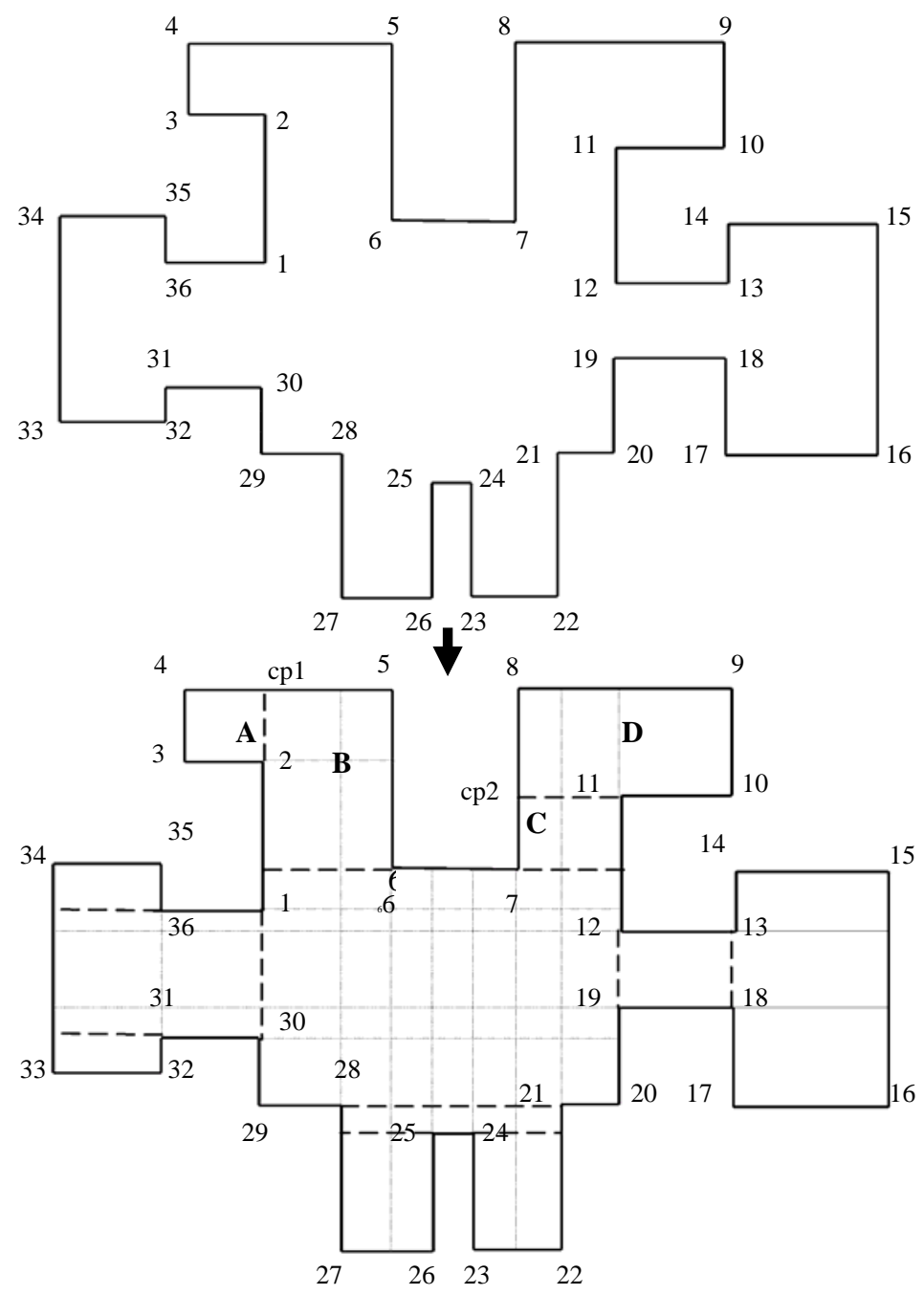

Fig.4 Upper : Building polygon is expressed as a set of its edges' turning direction; LLRRRLLRRRLLLRRRRLLRLRRLLRRLRLLRRRRL

Down : An orthogonal polygon with possible DLs( dotted lines ) 


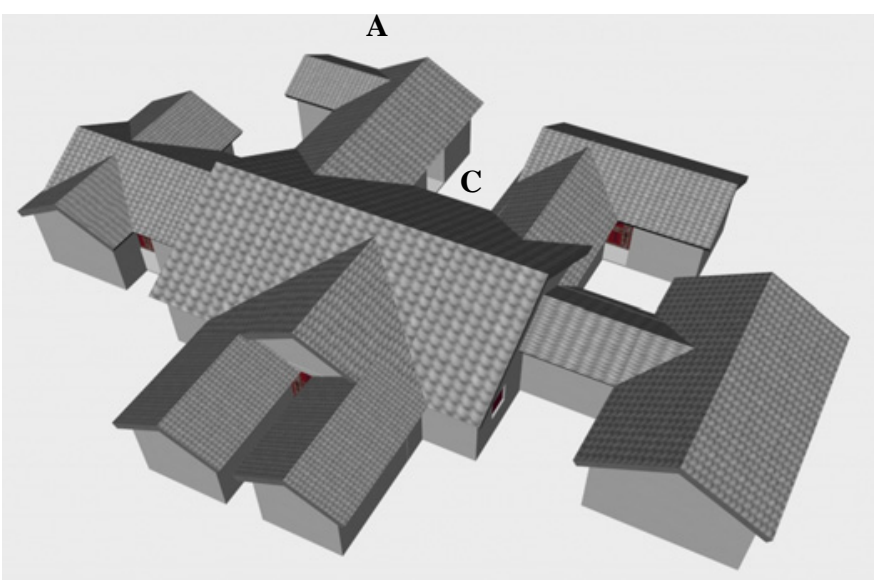

Fig.5 a 3D building model automatically generated from the polygon shown in Fig.4

to be implemented a partition.

1) A DL that cuts off 'one rectangle'.

2) Among two DLs from a 'L' vertex, a shorter DL is selected to cut off a rectangle.

3) A DL whose length is shorter than the width of a 'main roof' that a 'branch roof' is supposed to extend to.

Here, as examples shown in Fig. 5, a ' branch roof' is the roof that extends to a main roof and a 'main roof' is the roof that is extended by a branch roof.

Why we set up these conditions is that like breaking down a tree into a collection of branches, we will cut off along the thin parts of the branches of the polygon. So, we propose a scheme of prioritizing the DL that forms a branch roof, based on the length of the DL. Since each roof has the same gradient in most multiple-roofed buildings, a roof of longer width is higher than a roof of shorter width and 'probablemulti-roofed buildings' take the form of narrower branch roofs diverging from a wider and higher main roof. Narrower branch roofs are formed by dividing a polygon along a shorter DL and the width of a branch roof is equal to the length of the DL. For example, two DLs labeled A, B in Fig. 4 are drawn in a clockwise direction from vertex 2. Among two, a shorter DL A is selected so that four vertices 2, 3, 4, cp1 form a branch roof that will extend to the wider roof. On the other hand, two DLs labeled C, D are drawn in a counterclockwise direction from vertex 11. Among two, a shorter DL $\mathrm{C}$ is selected so that four vertices $\mathrm{cp} 2,8,9,10$ form a roof that will be extended by the narrower roof. Fig. 5 shows a 3D building model with the roof that extends to the wider roof and the roof that is extended by the narrower roof.

How the system is finding 'branches' is as follows. The system counts the number of ' $R$ ' vertices between ' $L$ ' vertices. If the number of ' $R$ ' vertices is two or more, then it can be a branch. One or two DLs can be drawn from ' $L$ ' vertex in a clockwise or counterclockwise direction, depending on the length of the edges of the polygon.

Fig. 6 shows partitioning process of orthogonal building polygon into a set of rectangles. In the process, the DLs that satisfy the conditions mentioned are selected to be implemented a partition. By cutting off one rectangle, the number of the vertices in a body polygon is reduced by two or four.

After partitioning, the edges' lengths and RL data are recalculated to find new branches. Partitioning continues until the number of the vertices in a body polygon is four. After being partitioned into a set of rectangles, the system places $3-D$ building models on these rectangles.

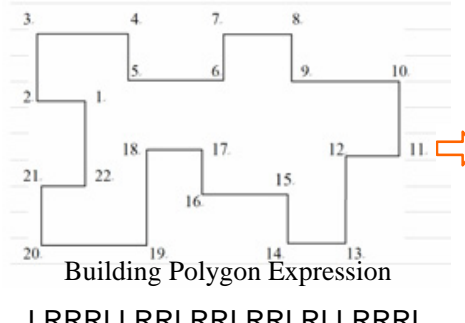
LRRRLLRRLRRLRRLRLLRRRL

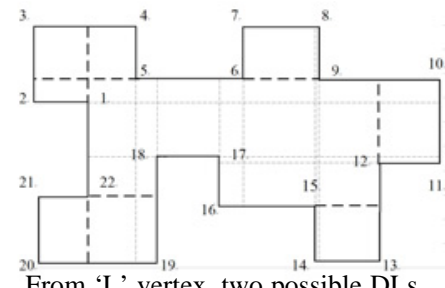

From ' $L$ ' vertex, two possible DLs can be drawn. The DL that cuts off a rectangle can be selected. 5

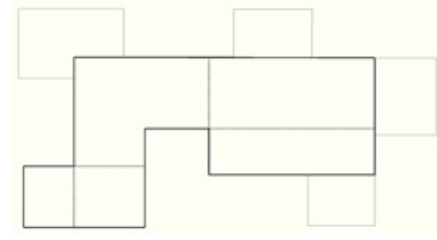

A DL that satisfies the conditions is selected to be implemented a partition.
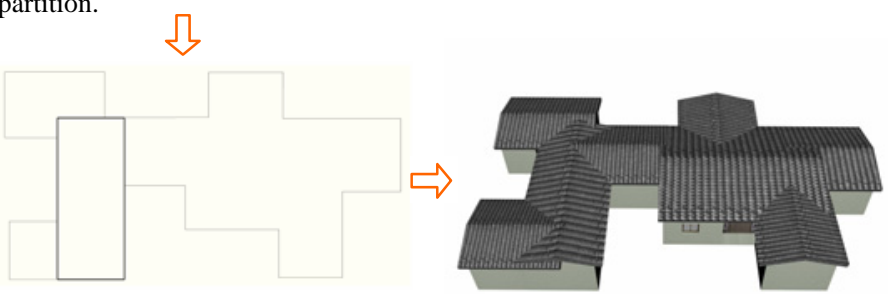

Division will continue until the number of vertices of a body polygon is four.

After division, 3D Building Models are automatically generated on divided rectangles bv using CSG.

\section{Fig.6 partitioning process of orthogonal building polygon}



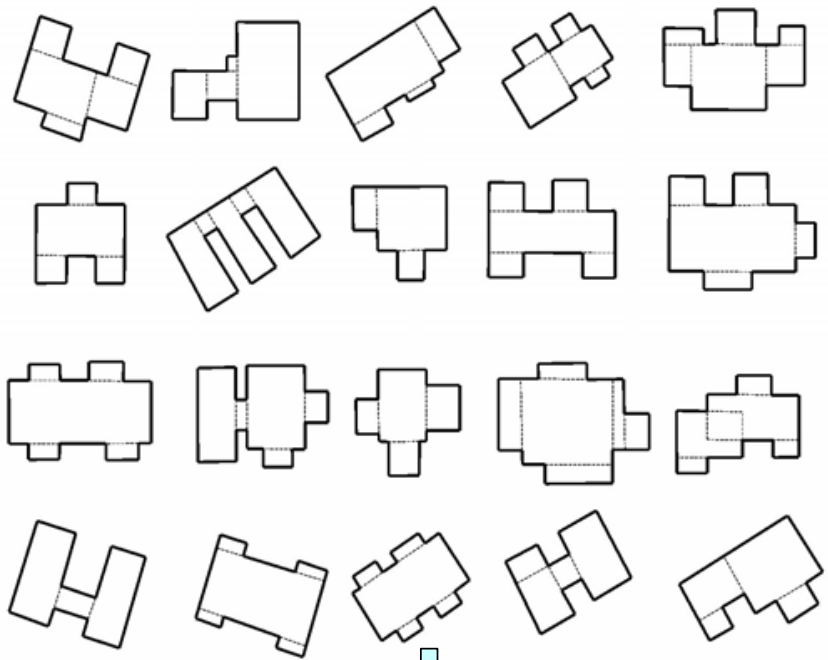<smiles>C1CCC(C2CCCCC2)CC1</smiles>
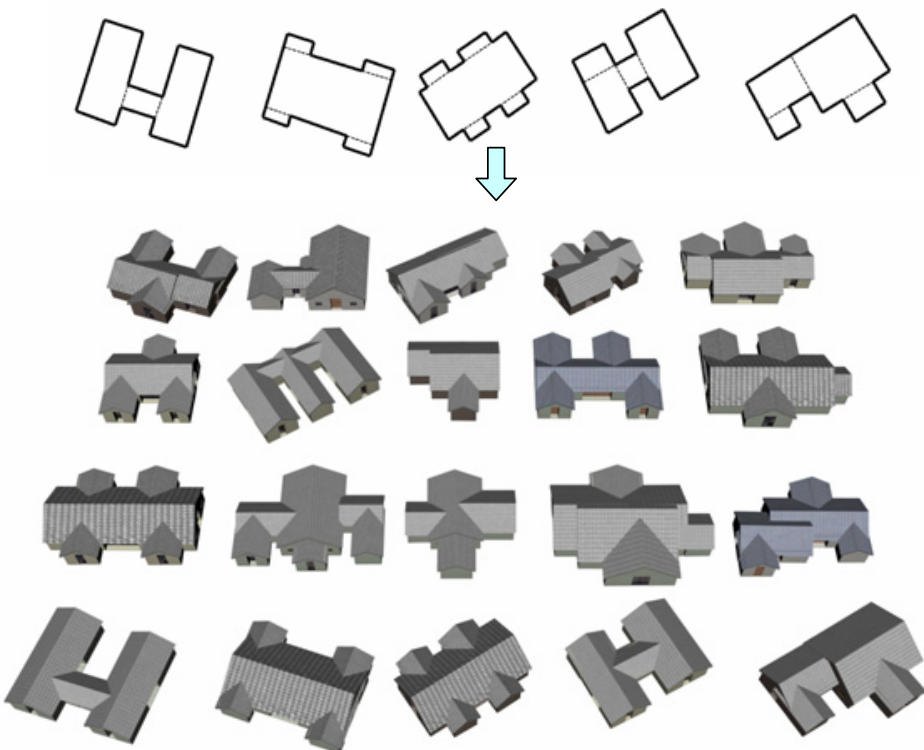

Fig.7 A variety of shapes of orthogonal building polygons with DLs implemented (upper)

3D building models automatically generated from divided polygons (below)

Fig. 7 shows a variety of shapes of orthogonal building polygons with DLs implemented and 3D building models automatically generated from partitioned building polygons.

\section{APPLICATION AND CONCLUSION}

Usually and traditionally, urban planners design the future layout of a town by drawing a map. So, it is convenient for urban planners who draw maps if the map can immediately be converted into a 3-D urban model. The system automatically generates a 3-D urban model so quickly that it meets the urgent demand to realize another alternative plan. Here is an example of a proposed 3-D urban model produced by the automatic generation system. Fig. 8 shows a proposed 3-D urban model where a variety of shapes of low-storied houses stand surrounded by tall buildings.
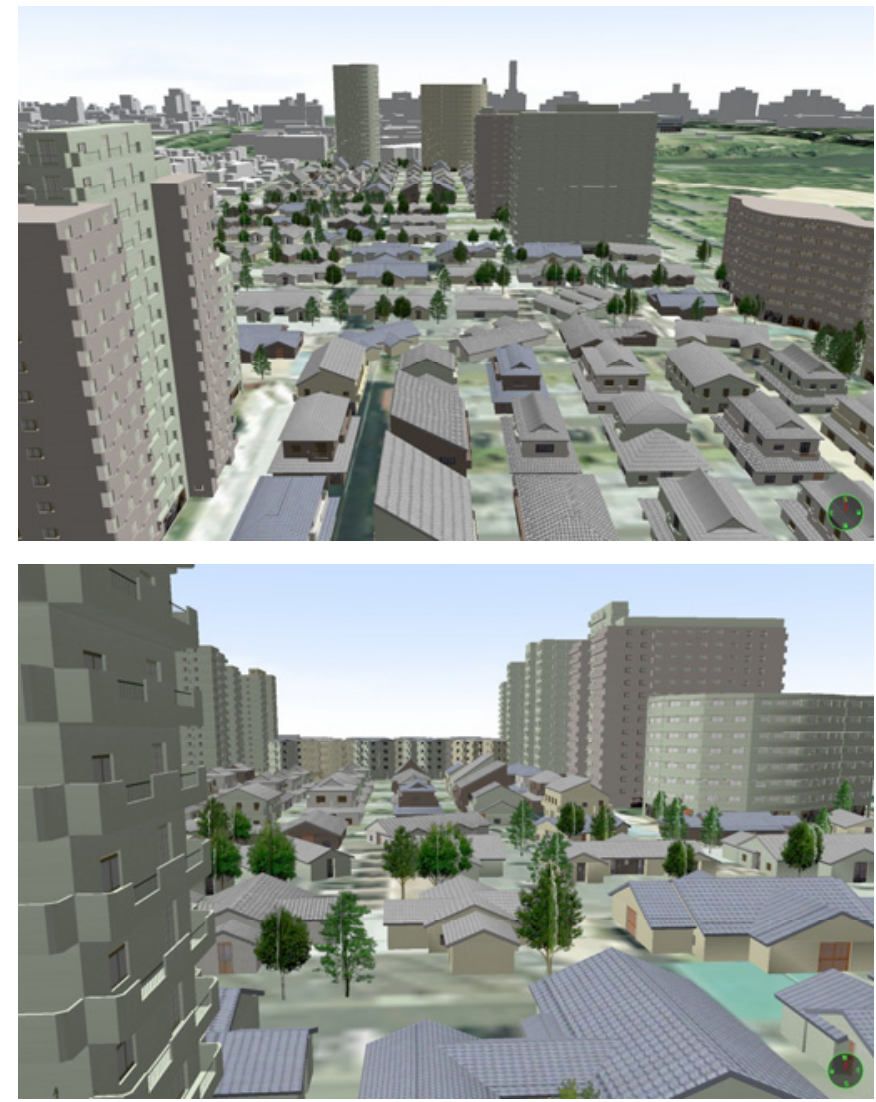

Fig.8 An automatically generated 3-D urban model from a digital residential map

In this paper, the CG and GIS integrated system that automatically generates a $3-D$ building model from orthogonal building polygons is proposed. By applying polygon partitioning algorithm, the system divides polygons along the thin parts of its branches. We propose a scheme of prioritizing the DL that forms a branch roof, based on the length of the DL. After breaking down into rectangles, the system is placing building parts on these polygons to form a 3-D building model.

\section{REFERENCES}

1) Kenichi SUGIHARA : Generalized Building Polygon Partitioning for Automatic Generation of 3-D Building Models, ACM SIGGRAPH 2006, Posters Session

2) Gruen, A. and Wang, X. (1998) CC Modeler: A topology generator for 3-D city models, ISPRS J. of Photogrammetry and Remote Sensing, 53, 286-295.

3) Gruen, A. and et al. (2002) Generation and visualization of 3D-city and facility models using CyberCity Modeler, MapAsia

4) Suveg, I. and Vosselman, G. (2002) Automatic 3D Building Reconstruction. Proceedings of SPIE, 4661, 59-69.

5) Fischer, A., Kolbe,T. H., Lang, F. (1997) Integration of 2D and 3D Reasoning for Building Reconstruction using a Generic Hierarchical Model, Workshop on Semantic Modeling for the Acquisition of Topographic Information from Images and Maps SMATI, 5/97, 1-21

6) Zlatanova, S. and Heuvel van den, F.A. (2002) Knowledge-based automatic 3D line extraction from close range images, International Archives of Photogrammetry and Remote Sensing, 34, 233 - 238 